\title{
Issues of reproductive health after child marriage: A study among NT-DNT community in Nashik district of Maharashtra
}

\author{
Rahul Ravindra Sarwade $^{1 *}$, Jagruti Hankare ${ }^{2}$, Trupti Chavan ${ }^{3}$
}

${ }^{\mathbf{1}} \mathrm{PhD}$ in Public Health, ${ }^{\mathbf{2}, 3}$ Master of Public Health in Social Epidemiology, ${ }^{\mathbf{1} 3}$ Tata Institute of Social Science, Mumbai, Maharashtra, India

\section{*Corresponding Author: Rahul Ravindra Sarwade}

Email: srahulrr@gmail.com

\begin{abstract}
Child marriage is common in community but remarriage are exceptional. Women's marriage, divorce, remarriage, sexual behaviour are all controlled by patriarchal norm living women subject to victimization. Women from this community suffer from not only discrimination based on their gender but also caste identity and consequent economic deprivation. Women's problems included gender and economic deprivation and social exclusion, which in turn results in the denial of their social, economic, cultural, and political rights. It is evident that the denotified and nomadic women and children being anaemic and live in unhygienic conditions which is confirmed by the field functionaries engaged in the development of the DNT community.

Out of 15 talukas in Nashik four villages were selected by purposive sampling and 200 women were selected for interview for quantitative part of study and six key informants, three Elderly women who perform delivery and eight tribal women were selected for interview for qualitative part of study. Tribal women gets married at very young age, and thus susceptible to have low knowledge and misconception about reproductive health which may leads to poor outcomes of the reproductive and sexual health. This study reveals that reproductive health knowledge of Nomadic tribal women in Nashik district is far below from satisfaction and lack of comprehensive knowledge of reproductive health prevalently noticed.
\end{abstract}

Keywords: Marriage, Divorce, Remarriage, Sexual behaviour, DNT.

\section{Introduction}

According to definition given by World Health organization, Reproductive health is a state of complete physical, mental and social well-being, and not merely the absence of reproductive disease or infirmity. In 1994, the international conference on population and development (ICPD) held at Cairo, Egypt, segmented prerequisite for human right framework to address reproductive health issues for poverty eradication. India was signatory to this agreement and had accepted reproductive health as essential for sustainable, economic and environmental development.

United Nations Population Information Network (POPIN), in its guidelines for Reproductive health states that, Reproductive health is right of both men and women which allow them to have access to safe, effective, acceptable and inexpensive methods of family planning. Also it is right of a women to have access to suitable health care services which would enable women a safe pregnancy and healthy childbirth. Reproductive health not only concerned about family planning, STD prevention and management and prevention of maternal and perinatal mortality and morbidity but also look after harmful practices, unwanted pregnancy, unsafe abortion, reproductive tract infections including sexually transmitted diseases and HIV/AIDS, gender-based violence, infertility problems.

Reproductive health disorders are responsible for to account for $5 \%$ and $15 \%$ of the overall disease burden. These are also make a major contribution to the global burden of disability, particularly for women, accounting for $21.9 \%$ of DALYs lost for women annually compared with only $3.1 \%$ for men (Murray \& Lopez, 1998). On an average, $40 \%$ of women, which accounts for 300 million pregnant women in a given time suffer from pregnancy related health problems and disabilities(Family Care International,1998). Further, estimated more than 529000 women die of pregnancyrelated causes each year (WHO 2006).

Tribal women are lacking in facilities of health care availability, accessibility and they are unaware about available health care facilities (Reddy 2008). Through NFHS, we succeeded to enhance and update our database. However, we failed to address some major reproductive health issues among women like morbidity of reproductive health have been neglecting since long time. We are still lacking in data regarding quality of care. Likewise, women's ability to exercise reproductive choice is again one factor, which we have not focused much (Jejeebhoy 1999). Poor knowledge and understanding of reproductive health problems is major issue in tribal population. Among various states where nomadic tribes are prevalent, where HIV cases are measured. Awareness and knowledge of AIDS among Indian tribal women had studied. National Institute of Medical Statistics (a body under ICMR) average 2.5 million people are living with HIV/AIDS, where women account for around one million. However with such an alarming context majority population don't even heard about HIV (NFHS-III, 2005-06). Over 30,000 ever-married women had surveyed about their awareness and knowledge of AIDS result showed very poor level of awareness of HIV. Among those, knowledge of transmission and prevention was very poor. Result also showed that rural, poorly educated, and poor women were the least likely to be AIDS aware and whoever were aware they found with poorest understanding of the syndrome (Balk and Lahiri 1997). In such way, various studies on reproductive health of tribal women revealed their poor understanding of reproductive health problems. 
Many women have perceptions that white discharge, painful menses it happens with everyone and such problems remain unfold (Jejeebhoy 2005). In rural India, many women not allow to cook or to worship during menstrual period (Jejeebhoy 2005). One study showed that 95\% women believe white discharge as most severe problem (Bang and Bang 1994). In Bangladesh, women consider their reproductive health problem and vaginal discharge because of internal body heat (Rose et al. 1998). These problems shows that the lack of awareness and false perceptions leads to major health illnesses. It is also observed that in a tribal population, culture and customs are seen with tribes influence the knowledge, beliefs and attitude toward reproductive health (Basu, 2000; and Salehin, 2012). India is hometown to almost more than half of the world's tribal population. The population of nomadic tribes in India is 150 million, approximately $15 \%$ of total Indian population (Ministry of tribes). On an average, there are 533 tribes were spread throughout various regions of India (swain, 2003) out of which 15 million in Maharashtra. Nomadic tribes have specific features because of the ever changing habitat also each tribe is unique in its culture, tradition, values, and believes. Nomadic tribes, on an account of their "Nomadic" and distinct mode of existence are at a disadvantage. For centuries, the tribal groups have remained outside the process of the general development (Ministry of Tribal Affairs 2010). They move from place to place in search of livelihood and hence deprived of benefits of citizenship in the republic as they don't have "fixed address of residence". In such a context, nomads face problem of registration as citizens, sequentially deprived of basic human needs. The deprivation and poverty continues in the community. Most of the nomads beg food, gather food, live in extreme unhygienic condition and drink any available water.

Various studies done so far found that health status of women is one of the significant determinant of maternal mortality (Global health action, 2013) reproductive health of nomadic tribal women is rarely addressed as it is considered as natural process. There is a general agreement that the health status of tribal population is very poor. Different studies have tried to establish this with the help of morbidity and mortality and health statistics. Tribal population have distinctive problem not because they have special kind of health but because of special placement in difficult areas and circumstances they live in (Naik et al). Santhya et al. (2008), carried out a survey among young men and women in two states of Madhya Pradesh and Andhra Pradesh in 2005 to 2007 and observed that the dominance of the men over the sexual relations do not allow their female partner to negotiate for safe sexual practices, This shows male dominance over female sexual behaviour in a community.

Due to the extreme poverty one who get most affected are women. These indigenous are malnourished (Samuel et al, 1992 Maiti et al, 2005) and their dietary energy intake is not adequate to compensate long and heavy work load. Along with that women does not seek medical facilities from health centres. They simply neglect their even serious health problems like RTI/STD, Pregnancy related complications, menstrual disorders.

\section{Materials and Methods}

The paper aimed to issue of reproductive health after child marriage among Nomadic Tribal Women of Nashik District of Maharashtra. This is a descriptive type of study. Quantitative method is used to assess socio-economic background, age at marriage, menstruation related knowledge and practices, unmet need of contraceptive, abortion and infertility related information, pregnancy and delivery details and Qualitative method is used to understand the respondents perception about services available for maternal health care and socio-cultural practices, belief system and health seeking practices among nomadic tribal women of Nashik District, Maharashtra. Among the target sample, 200 women were selected for interview for quantitative part of study and six key informants, three Elderly women who perform delivery and eight tribal women were selected for interview for qualitative part of study from each block the villages were selected by purposive sampling due to the higher concentration of nomadic tribal population. Four villages were selected from Nashik taluka, one villages from Niphad taluka, one village from Dindori and one village from Trimbak taluka. The tribal communities in the Nashik district selected for the sampling Pardhi, Joshi, Vaddar, Mariaiwale, Vaidu, Laman, Vanjari, Madari, Sayyed communities. For in-depth interview, the key informants, Traditional birth attendant (TBA) and community group leader and elderly women were selected. Majority of my target samples reside in the following place so purposively selected. In order to have health situation of NT and DNT community reasonable sample of 25 is taken irrespective of size of those communities The participant of the study adolescents and women who are married and belonging to nomadic tribes specifically in the reproductive period of their life. These women are in age group ranging from 12 to 49 years.

\section{Results and Discussion}

Age at marriage is a significant variable which determines reproductive health of women and men. In nomadic tribes average age at marriage is 12 to 16 years and majority of women got married in same age group. Nomadic tribes don't have idea about age. As child marriages have been prohibited by government communities have raised age limit to 12 to 15 years or according to menarche which is considered as benchmark for deciding age of marriage. It was noted that women in most of the nomadic tribal communities generally got married at a very early age. Women in tribal areas could not tell the age at which they marry, but said that usually they get married on commencement of menstruation or within two years of the onset of menstruation. Sometimes they decide the marriage or select the groom and wait for menarche to take place. The minimum age of marriage is as low as 8 years. Almost half of the population, 43.5 percent women married at the age below 14 years, which can be considered as child marriage. About $54.5 \%$ women married at 15 to 18 years of 
age and only $2 \%$ women married at age above legal age of marriage. Altogether there are $98 \%$ respondents who got married before 18 years. Mean age of marriage is $14.45 \%$. The elderly women agreed to the fact that, they get their children married just immediately after menarche. Reason quoted for this are poverty, culture, fear getting sexually abused.

Table 1

\begin{tabular}{|c|c|c|}
\hline $\begin{array}{c}\text { Age of Respondent } \\
\text { at the time of } \\
\text { marriage }\end{array}$ & Frequency & Percentage (\%) \\
\hline 08 to 14 years & 87 & 43.5 \\
\hline 15 to 18 years & 109 & 54.5 \\
\hline 19 and above & 2 & 2.0 \\
\hline
\end{tabular}

The problem of reproductive health of women in India is characterized by its ubiquitous silence. Reproductive health status of women is characterized by lack reproductive health knowledge and lack of autonomy of women in India. In general, poor knowledge and rigid patriarchal society creates difficulties for women to fulfil their reproductive health needs. Women from marginalized and vulnerable community like nomadic tribal population face additional difficulties to fulfil their unmet need of reproductive health. Tribal population of India is recognized as the marginalized section of the society which is characterized by prevalent low knowledge and misconceptions about health related matters. Tribal women get married at very young age, and thus susceptible to have low knowledge and misconception about reproductive health which may leads to poor outcomes of the reproductive and sexual health. Tribal women faces double burden of environmental difficulties and societal exclusion compared to other communities. Various data from national health surveys as well as many other research studies suggested that the reproductive health status of tribal women is poor as compared to the women from other community. Most of the earlier studies have focused HIV and contraception of tribal community however comprehensive efforts are essential to understand the reproductive health status of tribal women. The nature of patriarchal society creates difficulties for women in fulfilling their reproductive health needs. The low socioeconomic condition is associated with poverty, lack of awareness about personal hygiene, sanitation, health care \& nutrition and livelihood skills to increase productivity using local resources.

In reproductive health problems of women, menstrual health problems, RTI, abortion and contraception has been explored in this study. Respondents who were heard of health problems related to menstruation reported the symptoms related to menstrual problems, Menarche is occurrence of first menstrual period in females. Majority of respondent had menarche in the age group of 13 to 15 years. 5.5\% respondent had experienced it in 16 to 18 years of age group. Mean age of menarche $13.48 \%$. Further respondent were asked regarding problems suffered during last 6 months. Some $(31.5 \%)$ reported problem related to menstruation. Among those who had problem, 16.6 reported painful menstruation,
$1.38 \%$ reported frequent or short period of menses, $3.47 \%$ reported irregular menses while $2.08 \%$ have problem of scanty bleeding, $3 \%$ had experienced blood clots or excessive bleeding while $4.5 \%$ had white or other coloured discharge. Few respondent sought medical treatment for these disorders. They consider it normal and expected to occur sometime later in life. Practices related to menstruation $66 \%$ respondent use old washed cloths while only $4 \%$ use sanitary napkin and $3 \%$ use new cloths. Almost $15 \%$ of the respondent do not use any of the absorbent material during menses which they had experienced in the last three months. Around $25 \%$ of the women in same place of study region reported to have experienced menstruation related problems. Most prevalent problems reported by respondents were painful period, irregular periods and scanty bleeding. This result of the study was inconsistent with many other studies (Singh et al. 1999; Karthiga, 2011; Kulkarni and Durge 2011; Nair et al. 2012; Mohite et al. 2013) which reported, lower abdominal pain during menstruation as most frequent menstrual complaint. Majority of the women used cloths as menstrual napkins and. $15 \%$ of the respondents not even using any absorbent material, respondent were asked about their pattern of using material on first and second day of menstruation. One Respondent stated that absorbent material is not used by them. According to them there is no place to reside, the question storage of cloths is not valid. Her response regarding changing cloths was,

"Aamchyat kunibi asal vaparat nahi. Aamacha rahayacha thikana nahi mag kapde kuth sathavnar? Municipality yeun aamach saman fekun detyat, ithun haklun detat, samanach kam rahyal? (24 Years, female, Nashik)". There is always fear of leaving place due to pressure from municipality administrator, then it is difficult to store any stuff. Again privacy is the major issue. Open places where pal are situated privacy is serious problem for women, they have to get up early at 4-5 O' clock for bath. When asked regarding bath and hygiene during menstrual cycle, one respondent said, "Anghol "kay kay bayka kartat parda laun hitach, tith bathroomch pach rupay ghetyat an kapde dhuvayach dha rupay, mag kapad dhuvayala kuthun parvadnar?". This means only few women can manage to take bath on the open space with the help of cloths as a curtain in early morning. It is difficult for women residing here to spend five rupees for bathing and 10 rupees for washing clothes. Affordability bring restriction over hygiene and sanitation. Income affects adversely the use of bathroom and toilet facilities. A14 year old married girl from Pardhi community) said, "Aami asal kay nay vaparat, sadi na petikotach vaprato, an pali geli ki dhuvun takto." Which means all in our tribe don't use any absorbent material, what we do is let soak cloths (saree and petticoat) with blood and according to availability of money, go to bathroom for washing. Mostly they take bath after four to five days, after menstruation is over. A 13 year gajra vendor girl said, "Pali ali ki hitach basun rhav lagat, pais hatat astil tas angholila jato." Respondent tries to explain that whenever I have menses I have to sit at one place. If I would have money then I would go to have bath. Which suggest restriction laid by 
menses compel women to sit at a place for 14 to 15 hours a day for 3 to 5 days just to avoid mobility. While answering frequency of changing pads, respondent said that there is scarcity of drinking water, it if difficult to waste water for changing cloths, thus we use cloths and through them after use. These are those old cloths which they get from houses. Elderly key informant respondent from Madari tribe said, "ladki log lagate purana kapada, vaparneke baad fenk dete kachare me, pani kahaa hai dhone ke lie, yahaan pe pine ke pani ka vaanda hai'. This suggest that lack of water resource restrict the use of water for menstrual and personal hygiene. Their personal hygiene during menstrual period is very poor which is mainly due to lack of knowledge about the diseases that occur can occurred due to unsanitary condition, lower socio-economic status and the inaccessibility to sanitary pads. Though unlike Hindu culture there aren't social or cultural rituals which restricts women or seclude them but problem is with availability of bathroom or toilet, accessibility of clean and sufficient water are major issues. Not having toilet or secure place with adequate privacy for managing menstrual hygiene existed for many respondent at which would normally be changed around two to four times during menstruation. They usually take bath on fifth day of menstruation as bathrooms are not affordable to them. They wash their genitals with water only when they go to toilet which shows agony through which women has to go. Lack of water, absorbent material, lack of awareness about menstrual hygiene, community perception and taboos these are multiple factors which make situation complex. Though most of the women were experiencing their menstrual problems since long duration, very few women had discussed about it with husbands. As compared to other reproductive health problems like abortion, RTI, infertility, women had discussed less regarding menstrual health problems. The reason could be lack of privacy or stigma and their habit and belief to consider it as general problem. This becomes the most difficult task while educating the people. Ronald had discussed the same culture and beliefs in his article (Andersen 1995). Respondents who had received treatment for the problem of menstruation largely prefer the traditional healers followed by government hospital (PHC). This might be because tribal respondents were engrossed with superstitions. These people have faith in traditional healers who practice magical and religious rites. Along with this, traditional healers also use indigenous herbal treatment for the common symptoms of diseases. Similar situation had explored in one study where author had studied determinants of tribal health care in Chhattisgarh State. Study had suggested that the traditional folk medicine and health culture play a significant role in shaping tribal life (Balgir 2011). In studied population, majority of respondents were aware about the problem of difficulty in conceiving and $12.3 \%$ respondent had experienced problem in conceiving. Prevalence of problem in conceiving was reported for the first conception. Respondents had received treatment for the problem of difficulty in conceiving but they did not have knowledge about the treatment. Respondents were unaware about who exactly had problem, which was causing difficulty in conceiving. Majority of respondents received treatment from traditional healers followed by government hospital for, their problem of difficulty in conceiving.

Majority of respondents had heard about the abortion, but did not know exact knowledge about spontaneous and induced abortion. Around $23 \%$ respondents have experienced abortion, which is very high. Women don't have any source of information about abortion and appropriate places for abortions. Most common methods of contraception known to the respondents were female sterilization, followed by contraceptive pills and condom. Awareness of respondents found to be very low about male sterilization, IUD and emergency pills. Use of contraception found to be low negligible. Most prevalent method of contraception used by respondents was female sterilization followed by contraceptive pills and condom. Majority of respondents had utilized government health sector for contraception. Almost $90 \%$ admit that they don't have any idea about contraceptives and whatever they know is about female operation. A 22 years old respondent working as a construction worker who had given a still birth at 9 months pregnancy said, "Potat rahilel na navava mahinyat dactarla dakhival ter te mhanal por halat nahi tela baher kadhav lagel, te pani laun baher kadhal tar par jalal (maceration due to stillbirth) hot, konachi tar baa karni zali asal." A respondent had stillbirth at ninth month of pregnancy which required delivered with medical aid. As there was no fetal movements baby was macerated. But event is associated with supernatural powers and black magic. Women don't have any check-up before and after delivery, neither they have awareness of those check-up for wellbeing of baby and new-born. Result of this, they give adied children after going through pain for whole nine months, which is absolutely distress for women. A women working for telephone cables road digging work on contract basis, said, 'unha tanhach kama karav lagat bai, techyat potat kas rhail. Don vel pot khali zal mah gharatach. Kunala dakhvav. Garibacha kuth dactar? (29 Years, Vadar tribe) A women shared her experience of abortion twice in past. She had not visited any medical personnel and associate it with hard physical work. Also issue of poverty is also mentioned. It shows sheer agony of women who has to work hard irrespective of any whether. Though she is not able to have money which could be used for her abortion treatment. It is miserable condition where women can't access treatment doe to extreme poverty. It clearly indicate poor knowledge and awareness about contraceptives. Women wanted to use spacing methods but don't have any idea about how to get privilege of contraception. High fertility and subsequent deliveries made women suffer. Pregnancy related information-The wide range of population have not registered themselves in the institution for pregnancy. Number of visits to institute for ANC check-up are very less. Women hardly go to hospital. Hospitals are last option for complications during pregnancy. Less than 30 percent women had visited for any kind of ANC check-up during their last pregnancy. Though prevalence of complication is less in community, women are reluctant to visit even to get iron and folic acid supplementation. Due to extreme poverty, 
gender differential, cultural norms and lack of awareness about pregnancy there isn't concept of supplementary nutrition. Pregnancy is treated as natural phenomenon and women doesn't get any additional befit even at household level. As a result of this, women are malnourished and underweight. Home delivery is choice of delivery and hospital delivery are preceding options to complications. Most of the time deliveries are carried out by mother in laws and traditional birth attendant and measures adopted buy them are unethical and unhygienic. The abandoned mothers do deliveries of their own at times husband carries out delivery. It put light on the fact that community need assistance for safe motherhood, else life of women is on the edge of death. Those who have awareness about institutional delivery but have many barriers in accessing these services. Majority of population could not access due to expensive hospital treatment. $90 \%$ were reluctant to go to hospital as the environment is not comfortable and stay is long at hospital because of which families suffer. The influence of societal pressure is so much so family does not allow them to go to hospital. Even wide range of population have not realized benefits of institute delivery and thus they don't feel it is required to go to hospital during pregnancy. Sheer $14.2 \%$ women could receive post -natal care. 48 Hours post delivery are considered as crucial period. But none of the government scheme could reach these women. It is ironical that $70 \%$ women have to resume to her work immediately post - delivery. She hardly get 10 days rest after such exhaustive physiological process. Prevalence and awareness of RTI/STI. An attempt was made to assess whether respondents were aware of RTI/STI. Forty percent respondents were reported that they had heard of RTI. Information was collected on the common symptoms of reproductive tract infections and sexually transmitted infections from women. The prevalence of reproductive tract infections (RTI) and sexually transmitted infections (STI) is judged by their symptoms. $31 \%$ of respondents reported that they had experienced symptoms related to RTI. Low backache was the prevalent symptom reported by respondents followed by abnormal vaginal discharge, respondents reported, itching or irritation over vulva, significantly, also found vaginal discharge as the most frequent symptom of reproductive tract infection. Majority of respondents had not discussed about their problem of RTI with their husband. Respondents with vaginal discharge reported that vaginal discharge was wet, mucoid and with blood stain. Studies by khanna et al. (2005), Jain et al. (2009) and Nair et al. (2012) also found abnormal vaginal discharge as the most frequent symptoms of reproductive tract infection. Majority of respondents recorded their source of information for RTI. Husband and relative were found to be major source of information for the high proportion of respondents. Proportion of respondents reported for the print media as source of information was very low; it might be because of illiteracy they are not convenient with print media. In case of the presence of at least one symptom, healthseeking behaviour of respondents was reported for RTI/STI.

\section{Conclusion}

Reproductive health of women is important indicator for the good health status of women. This study reveals that reproductive health knowledge of tribal women in Nashik district is far below from satisfaction and lack of comprehensive knowledge of reproductive health prevalently noticed. Reproductive morbidities found to be more prevalent among tribal women of study area. Mean age of marriage is 14 years for female and 17 years for men, both less than legally defined marriage age. Total fertility rate, high early marriage, successive pregnancies accompanied with low calorie of food intake and inaccessibility, and underutilization of medical facilities lead to high maternal morbidity and mortality rate. 'Maternal depletion' is thus the result of early mating, continuous cycles of pregnancy.

More than half of the women reported with one or more symptoms related to menstruation and RTI. Among these respondents, pain in abdomen related to menses, abnormal vaginal discharges, low backache and genital itching and boils over vulva are the main reproductive morbidities reported during this study. With this scenario, more than two third of women did not seek any treatment. Hence, their treatment seeking behaviour has been found to be very poor. Very significant difference has been found in awareness and use of contraception normal thing. This makes delay in diagnosis and treatment of diseases. Due to the geographical constraint, respondents could not seek treatment easily. To travel to PHC and district hospital become very difficult through this hilly area, therefore patient mostly prefers traditional healer first as the service is readily available in village. For the first step, respondents in this study area approach to traditional healers and then if disease not get cure they approach to the government hospital. While concluding the topic paper would like to mention there is lot of scope to improve reproductive health of women through health education. There are some socio-economic and social determents, which are deeply rooted in community and responsible for the vulnerability of women. By addressing these determinants, it is possible to improve reproductive health of women in study area.

\section{Source of funding}

None.

\section{Conflict of interest}

None.

\section{References}

1. Anderson, J. (1973). Health Services Utilization: Framework and Review europepmc.org/articles/pmc1071757/pdf/hsresearch005640011

2. Bokil M. De-Notified and Nomadic Tribe: A Perspective, Economic and Political Weekly, 2002;37(2):148-15.

3. Chauhan P. Maternal mortality among tribal women as per gravidity at a tertiary level of care in baster Chhattisgarh, India. Int J Biol Med Res 2012;3(1):1377-84.

4. Ekka N. Impact of modernization on tribal religious customs and traditions: a case study of Rourkela. 2013. 
5. Freedman L. (2004). Interim Report of Millennium Project Taskforce 4 on Child Health and Maternal Health. New York: UNDP.

6. Fuchs S. (1979). Aboriginal Tribes in India. Delhi: Macmillan India Ltd.

7. Gaikwad L. (1987). Uchlya (Marathi). Shree Prakashan Pune.

8. Galaty. (1981).The world of Pastoralism, The Guilford Press, New York and London.

9. Glacier. Sexual and reproductive health: a matter of life and death, Lancet 2006;368:1595-607

10. Glacier A, Ahmet. Sexual and reproductive health: call for papers, 2005;366.

11. http://www.epw.in/journal/2007/40/editorials/denotified-andnomadic-tribes-nowhereexistence.html\#sthash.rOZ9dC3x.dpuf.

12. http://www.thehindu.com/thehindu/mag/2003/07/06/stories/20 03070600770100.htm..(vaidu)

13. Jayakrishnan. Health problems of construction workers. Int $J$ Med Public Health 2013;3(4).

14. Jejeebhoy, Sandhya. (2011). Sexual, reproducyive health of young people in India: A review of policies, law and programmes.

15. Langer A. Cairo after 12 years: successes, setbacks, and challenges. Lancet, 2006;368.

16. Lashkar V. Denotified and Nomadic Tribes of Maharshtra in India. Int J Ideas, 2012;26(309).

17. Mahapatro S. Utilization of maternal and child health care services in India: does women's autonomy matter? J Family Welf. 2012;58(1).

18. Mahatribal.gov.in. DOI: 02/03/2017. Tribal Development Commisionarate, Tribal Development Department, Government of Maharashtra.

19. Majumdar, N. (1944). The Fortunes of Primitive Tribes. Lucknow: Universal Publishers.

20. National Commission for Denotified, Nomadic and Seminomadic tribes. Ministry of Social Justice and Empowerment, Government of India, Report, Vol.1, 2008.

21. Newman L. Global Estimates of Syphilis in Pregnancy and Associated Adverse Outcomes: Analysis of Multinational Antenatal Surveillance Data. PLoS Med 2013;10(2):e1001396. doi:10.1371/journal.pmed.1001396

niti.gov.in/content/maternal-mortality-ratio-mmr-100000-livebirths. DOI: 02/03/2017.

22. Pachauri, S and Subramanian S. Management of Reproductive Tract Infections in Women: Lessons from the Field, Implementing a Reproductive Health Agenda in India: 1998;435-505.

23. Prashant. Determinants of Maternity care services utilization among married adolescents in rural India. Preventing low birth weights, National law academics, Institute of Medicine, 1985.

24. Radhakrishna M. Colonial Construction of a 'Criminal' Tribe: Yerkulas of Madras Presidency, Economic and Political Weekly. 2000;35;(28):2553-63.

25. Rathod, M. "The Denotified and Nomadic Tribes Rights Action Group Newsletter", DNT Rights Action Group 6 United Avenue, Near Dinesh Mills, Vadodara, India.

26. Ray S, Dasgupta A. Determinants of menstrual hygiene among adolescent girls: A multivariate analysis. Natl J Community Med.

27. Reddy S. Health of tribal women and children-: An interdisciplinary approach. Indian Anthropologist. 2008;38(2):61-74

28. Reproductive health in India (1994). Forum for Women's Health. Womens Health Newsl.

29. Reproductive health in India, (1994). Forum for women's health, National library of medicine, National Institute of health.
30. Sabharwal, N and Sonalkar, W. (2015). Dalit Women in India: At the Crossroads of Gender, Class, and Caste Global justice: theory practice rhetoric.

31. Salehin M. (2012). Reproductive health of tribal populations in India: A sustainability approach, the University of Texas at Arlington.

32. Salehin M. (2012). Reproductive health of tribal populations in India: a sustainability approach.

33. Sexual \& reproductive health: http://www.unfpa.org/sexualreproductive health\#sthash.dqdiCV9f.dpuf

34. Shweta. (2004). Health and Social Welfare of De-notified and Nomadic Tribes in India).

35. Shweta. An intense blind spot of gender and health In India three times the size of Nordic Countries: health \& reproductive health Concerns of denotified and nomadic tribe women \& girls. Indian Streams Res J 2014;4(2).

36. Singh, B. (2012). Intervention of female identity in denotified tribes.

37. Tarafder T. Reproductive health beliefs and their consequences: a case study on rural indigenous women in Bangladesh. Aust J Reg Studies 2014;20(2):351.

38. The Millennium Development Goals Report (2015). The United States Government Global Health Initiative: Strategy Document,

39. Unicef. (2008) State of the World's Children 2009. New York.

40. United Nations News Center, 2010.

41. Vinitha C, Singh S, Rajendran A. Health and Population Perspectives and Issues: level of reproductive health awareness and factors affecting it in a rural community of south India. 2007;30(1):24-44.

42. Voices of DNT/ NT communities in India. (2016). Government of India.

43. Vora S. Maternal Health Situation in India: A Case Study. $J$ Popul Nutr 2009;27(2):184-201.

44. www.mapsofindia.com > Maps > Maharashtra Map > Districts. DOI: 01/03/2017.

45. www.unfpa.org/maternal-health. DOI: 02/03/2017.

46. www.shodhganga.inflibnet.ac.in/bitstream/10603/2019/1 